Business and Economics Research Journal

Volume 7 Number 12016

pp. $1-19$

ISSN: $1309-2448$

DOI Number: 10.20409/berj.2016116801

\title{
A Study of Nonlinear Dynamics in Equity Market Index: Evidence from Turkey
}

\author{
Riza Emekter ${ }^{\mathrm{a}}$
}

\author{
Benjamas Jirasakuldech ${ }^{\mathrm{b}}$
}

\begin{abstract}
The dynamics of Istanbul Stock Exchange (ISE) 100 index is explored in this study for the past 25 years. The main motivation of this paper is to find out the source and nature of any dependence in the ISE index. There is dependence in the log returns of the ISE. This dependence is not a linear dependence since no ARIMA models remove the dependence. Moreover, the dependence cannot be explained by nonlinear autoregressive process (GARCH) and important relevant macroeconomics variables. The persistence in the return dependence is not short term (3-months or less) in nature. Nonlinearity in the ISE index is caused by non-Gaussian innovations and it is not likely to be caused by chaos. Duration dependence test suggests that there is no evidence of a rational bubble in the log returns. There is some evidence of a structural break in the Turkish equity market around May 2000. However, the results do not change significantly when the same analyses are applied on the pre-and post-May 2000 periods. These results suggest that ISE 100 index is relatively efficient. Although there is dependence, the predictable component of the index is nonlinear, non-chaotic, and bubble-free. The predictable component is uncorrelated with any macro factors and cannot be explained by conditional autoregressive variance.
\end{abstract}

Keywords: Nonlinearity, Chaos, Turkish Equity Market, Market Efficiency, Random Walk

JEL Classification: G12, G14, G17

\section{Introduction}

Successful modeling of asset prices is very crucial for investors. It has been shown in a vast number of studies that asset prices exhibit non-linear structures (Hsieh, 1991; Lim and Brooks, 2011; Cariani, 2014). The non-linear dynamics in financial assets are of critical concern because they are inconsistent with market efficiency. In addition, using a linear model to price securities may result in an incorrect asset price and exploitable profit opportunities. Most of the recent empirical literature has focused mainly on discovering whether financial markets are characterized by non-linear behaviors. However, understanding the nature and function of non-linear dependence driving the market price has become increasingly more important in implementing a successful and accurate forecast leading to a profitable trade.

The purpose of this study is to examine whether the Istanbul Stock Exchange (ISE) stock markets are governed by non-linear dynamics. The popularity of the ISE equity index has been increasing in proportion to the rapidly growing economy of Turkey. Approximately 60 percent of the securities traded in

\footnotetext{
a Ph.D., Associate Professor of Finance, Department Head Robert Morris University, Department of Finance, Pennsylvania, USA, emekter@rmu.edu

${ }^{b}$ Ph.D., Professor of Finance, Slippery Rock University, School of Business, Pennsylvania, USA, b.jirasakuldech@sru.edu
} 
the ISE are owned by foreign investors. Therefore, the equity market in Turkey has become more important for global investors as well.

This study aims to answer two important questions about the ISE index: First, is there a persistent dependency in the equities traded in the Istanbul Stock Exchange? Second, if the dependency persists in the ISE equity index, what is the form of the dependency? In other words, is the dependence due to linear, non-linear, or chaos dynamics? This paper evaluates the non-linear dynamics of the ISE 100 index using rich variety techniques, including the BDS test, the chaos test, the Markov Chain test, the time reversibility test, the and duration dependence test.

There have been an extensive number of studies carried out to reveal non-linear structures in financial time series. In addition, some studies specifically analyzed the ISE for non-linear dependence and chaotic structures (Ozer and Ertokatli, 2010; Ozkaya and Ozkaya, 2012; Odabasi et.al., 2004). This study will make significant contributions to the current literature because none of the studies were as systematic and comprehensive as this study in analyzing dependence issues step by step. In the first step, a BDS test is applied to the log ISE index return series to diagnose for any form of dependency ${ }^{1}$. The BDS result reports evidence of dependence in the ISE returns. When several ARIMA models are fitted into the ISE time series and the residuals are tested again for dependence, dependence continues to persist. In the next step, some nonlinear models are employed to further investigate whether nonlinearity could possibly be one of the forms of dependency. When the ISE return is modelled as one significant GARCH term and one threshold order, the BDS test fails to reject the presence of dependency. Further K-map and Z-map analysis shows that the dependency in the ISE return is not caused by erratic and chaotic behavior in the Turkish equity market. A Markov chain test shows that the dependence is not a short-term dependence. A time reversibility test further provides supporting evidence that the dependence in the ISE 100 index is nonlinear and the non-linearity comes from the asymmetric innovations. Lastly, in accordance with the duration dependence test, the non-linear dependence evident in the ISE return is not consistent with the characteristic of a rational bubble.

The remainder of the paper is organized as follows. In the next section, we review the literature for nonlinear dependency in security prices. This section also provides a comprehensive review of stock returns behavior in Turkey. Section 3 describes our data and summarizes the descriptive statistics of the ISE 100 index and three macro-economic variables including Turkish Lira/\$ exchange rate, interbank overnight rate, and inflation in Turkey. In section 4, we present the descriptions of the BDS, K-map and Z-map analysis, Markov chain, Time reversibility, and Duration dependence methodologies in details. The empirical results are also presented in this section. Section 5 offers some concluding remarks.

\section{Literature Review}

There is a vast published empirical literature in non-linear dependence in the time series. The literature in this area focuses mainly on the techniques most commonly used to detect the dependency. Two popular techniques cited by many nonlinear studies are the BDS test developed by Brock et al. (1996) and the bispectrum test developed by Hinch (1982). Although the BDS test is one of the most powerful tests used to detect the dependence in a financial time series, it does not reveal whether the dependence is linear, nonlinear, or chaos in nature.

There are some studies specifically designed to determine the nature of the dependency. Several different versions of autoregressive conditional heteroskedasticity models (ARCH) originally developed by Engle (1982) are used to explain nonlinear dependence in financial time series. Hsieh (1989) and Bollerslev (1986) are among early studies that adopt the ARCH type model in examining the nature of nonlinear dependence. The regime switching model is the other popular technique commonly used to detect for nonlinear dependence. One of the earliest regime switching models can be attributable to the pioneering works of Hamilton (1990) and Teräsvirta (1994).

A chaos test is used to determine whether the dependence is caused by an erratic and chaotic dynamic process in the time series. Hsieh (1991) is one of the earliest studies that attempt to examine the 
chaotic behavior of the U.S. stock market. His study shows that the dependence in the U.S. stock index is not due to chaotic structures or regime changes, but it can be attributed to conditional heteroskedasticity. Subsequent studies that employ the chaos tests with Lyapunov exponents include Nychka et al. (1992) and Shintani and Linton (2004). Although the issue of nonlinear dependency was investigated extensively by many studies, empirical results from these large numbers of studies are mixed. In their survey of the literature of the evolution of stock market efficiency, Lim and Brooks (2011) conclude that most empirical studies find nonlinearity in stock prices in different countries with different market structure mechanisms. On the other hand, a large number of others studies find that stock market indexes are generally characterized by long lasting pure noise processes followed by short-lived nonlinear dependence. Lim et al. (2008) report similar stock market characteristic for ten Asian emerging markets. The study by Bonilla et al. (2006) also shows that equities in Latin America exhibit the pure noise processes, which last for a long period of time, and are followed by nonlinear dependence of a shorter duration. Similar stock market characteristics in Taiwan are also reported by Ammermann and Patterson (2003).

Although the issue of non-linearity in the equity market was studied extensively, the results were inconclusive. One interesting fact found by the study of Cariani (2014) can be used to settle these conflicting results in the literature. His study concludes that the higher the frequency of the data, the higher the likelihood to find nonlinearity in financial and economic time series.

\subsection{Nonlinear Studies on Istanbul Stock Exchange}

There are a number of studies to specifically unearth the nature of dependence in the Istanbul Stock Exchange (ISE) indexes. Some studies attribute the dependence in the ISE 100 to the chaos process (Ozer and Ertokatli, 2010; Ozkaya and Ozkaya, 2012). Other studies show that the nonlinear dependence in the Turkish equity market can be explained by autoregressive conditional heteroskedasticity (Harris and Kucukozmen; 2001; Bildirici and Ersin, 2009). Bali et al. (2008) further report that equity market in Turkey can be characterized by a significant higher mean reversion, particularly during the large falls of the market. Lastly, Ozun (1999)'s study shows that the dependence in the ISE is caused by the irrational behavior of investors.

Many studies that focus on examining the return behavior of the ISE index document an improving trend for the efficiency of the Istanbul Stock Market. These studies find non-linear dependence and/or chaos in the late $80 \mathrm{~s}$ and the early 90 s of the first period of the establishment of the Istanbul Stock Exchange, but fail to detect similar structures in later years, especially towards the end of 90s (Antoniou et al., 1997; Odabasi et al., 2004). Similar to the findings of Lim et al. (2011), Erkus and Ugur (2014) find that the ISE index can be characterized by random noise process for most of the period, followed by a brief chaotic period caused by several major national and international events.

A broad spectrum of methodology is employed in testing for non-linear dependence in the Turkish equity markets in various studies. Boyacioglu and Avci (2010) use an adaptive network-based fuzzy inference system to predict stock market return in Turkey. Many studies employ the Lyapunov Exponent to model the nonlinear behavior in Turkey; a few of these studies are Ozkaya and Ozkaya (2012) and Ozer and Ertokatli (2010). Lastly, the Hinich Bispectral tests are also widely conducted in many nonlinear studies of the Turkish stock markets (Ozer and Ertokatli, 2010; Yilanci, 2012; Erkus and Ugur, 2014).

This study will enhance and expand the current literature by employing the most systematic approach in exploring the non-linear dynamics of the Istanbul Stock Exchange 100 index. 


\section{Data}

The data consists of the monthly price index of the ISE 100 stocks quoted on the Istanbul Stock Exchange over the period from January 1988 to July 2013. The total observations are 306 . The continuously compounded monthly return of the ISE 100 is computed as, $r_{t}=100 * \ln \left(P_{t} / P_{t-1}\right)$, where represents the closing ISE 100 index value at the end of the month and $P_{t-1}$ is the prior month closing value. Three macro-economic variables in Turkey employed in this study: consumer price index (CPI), Turkish Lira/\$ exchange rate (EXRATE), and overnight interbank rates (INT). Consumer price index and exchange rate series are also obtained for the same period. Overnight interbank rate is used as a proxy for interest rate in Turkey and the data starts from January 1990, which includes 282 observations. All the data is obtained from the Bloomberg. Although the primary focus of this paper is investigating the nonlinearity in the ISE 100 returns, we use consumer price index, exchange rate, and interest rate as the macroeconomic factors that can potentially explain the variance of the ISE stock index. These three macroeconomic variables are used in the chaos tests to examine whether past nonlinear ISE100 returns and macro-economic variables exhibit stable or chaotic effects on future ISE returns. The logarithmic differences of three macroeconomic variables are constructed to render growth rate and stationary in the series. These macroeconomic variables are generally known to exert a strong influence on the securities returns, as shown by many studies (Chen, Roll, and Ross, 1986; Chen and Tzang, 1988; Lu and So, 2001).

The general statistical characteristics of each data series are presented in Table 1. The average monthly return of stocks on the ISE 100 index is $2.98 \%$. During the past 25 years, the ISE 100 returns yield a maximum return of $5.87 \%$, a minimum return of $-49.48 \%$, with a volatility of $14.40 \%$. Among the three macro-economic variables, interbank overnight rate varies significantly from the high of $355.00 \%$ to a low of $1.50 \%$. The average interbank overnight rate is about $44.71 \%$. The percentage change in Turkish/\$ exchange rates has a minimum of $0.001 \%$ and a maximum of $1.93 \%$ with a mean of $0.79 \%$. For Turkish inflation, the average inflation rate is $2.97 \%$ and the highest inflation rate was $24.71 \%$. Over the period study, Turkey has experienced the deflation rate of $6.23 \%$. When comparing the volatility among the three macro-economic variables, Turkish Lira/\$ exchange rate exhibits the least volatility of $0.71 \%$ when compared with the volatility of $37.99 \%$ of interbank overnight rate and $2.95 \%$ of inflation in Turkey. Apparently, all the data set is only mildly skewed but has a high degree of kurtosis. According to JarqueBera statistics, significant deviations from normality are evident.

Panel B of Table 1 reports the Ljung-Box (1978) Q statistics. The null hypothesis of no autocorrelation is rejected at lags 6 and 12 at the 1\% traditional significant level for the ISE 100 returns and all the three macro-economic variables. This is an indication of the serial dependence in the higher moments in the ISE returns. The result of augmented Dickey-Fuller (ADF) unit root is also reported in Panel $\mathrm{C}$ of Table 1. According to the unit root tests, the ISE 100 index level is non-stationary. However, when the ISE index is transformed to log return, the null hypothesis of a unit root is rejected at the $1 \%$ significant level. Similarly, Turkish Lira/\$ exchange rate series has the unit root but the first difference of the series is stationary. Both of the Turkish interbank overnight interest rates and inflation series are trend stationary. All further tests are carried out on stationary series.

We further tested whether there is any structural break in the ISE 100 index series. The Bai-Perron multiple breakpoints test is applied. The results show no evidence of structural break-points in the data. However, when implementing the Chow breakpoint test and Quant-Andrews breakpoint tests, evidence of a structural break in Turkish stock market is found and as shown in Panel D of Table 1 with the F-statistic of 6.2656. During the period investigated, the Turkish stock index experienced a structural break in the fifth month of year $2000^{2}$. 
R. Emekter - B. Jirasakuldech

Table 1. Summary Statistics of Monthly Real Returns of ISE 100 Index, Turkish Lira/\$ Exchange Rate, Interbank Rate, and Consumer Price Index

\begin{tabular}{lrrrr}
\hline \multicolumn{1}{c}{ ISE100 } & $\begin{array}{c}\text { Turkish Lira/\$ } \\
\text { Exchange Rate }\end{array}$ & \multicolumn{1}{c}{$\begin{array}{l}\text { Interbank } \\
\text { Rate }\end{array}$} & $\begin{array}{r}\text { Consumer Price } \\
\text { Index }\end{array}$ \\
\hline \multicolumn{1}{l}{ Panel A: Summary Statistics } & & & \\
\multicolumn{1}{l}{ Observations } & 305 & 306 & 282 & 306 \\
Mean & 2,9813 & 0,7927 & 44,7137 & 2,9749 \\
Median & 2,4558 & 0,6662 & 44,5000 & 2,3800 \\
Maximum & 5,8664 & 1,9281 & 355,0000 & 24,7100 \\
Minimum & $-49,4840$ & 0,0011 & 1,5000 & $-6,2300$ \\
Std. Dev. & 14,3991 & 0,7066 & 37,9960 & 2,9517 \\
Skewness & 0,3812 & 0,0601 & 2,6722 & 1,8916 \\
Kurtosis & 4,9050 & 1,2354 & 20,1008 & 12,9819 \\
Jarque-Bera & 53,5063 & 39,8872 & $3.771,7590$ & $1.452,8770$ \\
Probability & $(0.0000)$ & $(0.0000)$ & $(0.0000)$ & $(0.0000)$ \\
& & & & 910,32 \\
Sum & 9,0929 & 242,5645 & $12.609,25$ & $2.657,26$ \\
Sum Sq. Dev. & $6,30 \mathrm{E}+00$ & 152,267 & $405.677,40$ & \\
\hline
\end{tabular}

Panel B: Autocorrelation Test

\begin{tabular}{|c|c|c|c|c|}
\hline Ljung Box (Q6) & $5.2142^{* * *}$ & $1,757.0^{* * *}$ & $661.71^{* * *}$ & $366.27^{* * *}$ \\
\hline Ljung Box (Q12) & $10.4550^{* * *}$ & $3,362.3^{* * *}$ & $1232^{* * *}$ & $739.2^{* * *}$ \\
\hline
\end{tabular}

Panel C: Unit Root Test

\begin{tabular}{|c|c|c|c|c|}
\hline ADF Test Statistics & $-15.9468^{* * *}$ & $-15.9811^{* * *}$ & $-16.6085^{* * *}$ & $-17.3301^{* * *}$ \\
\hline
\end{tabular}

Panel D: Structural Break Test in ISE 100 Index

Sequential F-Statistic Determined Breaks: 0

\begin{tabular}{cccc}
\hline Break Test in ISE 100 Index & F-Statistics & Scaled F-Statistic & Critical Value \\
0 vs. 1 & 6,2656 & 6,2656 & 8,58
\end{tabular}

Notes: The monthly ISE 100 returns are continuously compounded. The study period for ISE 100 index, Turkish Lira/\$ exchange rate, and consumer price index is from January 1988 to July 2013. The study period of Interbank overnigh rate from Turkey is from January 1990 to July 2013. Mean, minimum, maximum, and standard deviation of ISE 100 returns are expressed in percent. Asymptotic standard errors of coefficient of skewness is $[24 / \mathrm{N}]^{1 / 2}$. Jarque-Bera test statistics test for departure from normality. $\mathrm{Q}(6)$ and $\mathrm{Q}(12)$ are the Ljung-Box Portmantaeu test statistics identifying the presence of sixth and twelve-order autocorrelaiton. Under the null hypothesis of no autocorrelation, it is distributed as c2 with 6 and 12 degrees of freedom, respectively. P-values are in parentheses. * indicates significance at the $10 \%$ level, ** indicates significance at the $5 \%$ level, and $* * *$ indicates significance at the $1 \%$ level.

\section{Methodology}

While earlier statistical test results provide evidence in favor of serial correlation and non-random walk behavior, we further empirically test whether such dependency can be explained by nonlinear, chaotic dynamics or bubble-like structures in the Turkish stock market data. Along with the rich and expanding nonlinear statistical techniques, we employ a systematic approach in investigating the nonlinearity. First, the Brock-Dechert-Scheinkman (BDS) test is implemented to see if there is any dependency in the ISE 100 
stock index. However, the rejection of the null hypothesis of independently and identically distributed (i.i.d.) series by the BDS test provides very limited information regarding the actual type of dependency inherent in the financial time series data. To further uncover the nature of dependency, the ISE 100 stock market is first modelled as a linear ARIMA and then non-linear GARCH model. Using the BDS test, the residuals from the fitted models are tested for dependency to identify whether it is linear or non-linear. Subsequent application of the K-Map and Z-map model is conducted to test whether the nonlinear stochastic dependence in Turkish stock market is consistent with chaos form. In complement with the BDS test, a Markov Chain test is also conducted to test for non-linear dependence. A time reversibility test is then applied to detect the mechanism generating the nonlinearity. The time reversibility test helps reveal whether the non-linear dependence is due to the functional form or due to non-Gaussian error terms. Lastly, a duration dependence test is performed to investigate whether the nonlinearity inherent in the ISE index can be characterized by a presence of rational speculative bubbles. With the implementation of the battery of general and specific nonlinearity tests on the ISE index, the observed characteristic of nonlinearity in the Turkish stock market can be substantiated to the extent that this feature is a stylized fact of stock market data.

\subsection{The BDS Test}

The BDS test developed by Brock, Dechert, Scheinkman, and LeBaron (1996) is a powerful test in detecting dependency in many financial time series. Prior studies that employed the BDS test and reported evidence of nonlinear serial dependence in the stock market data were overwhelming (Lim et al., 2006; Frank and Stengos, 1989; Newell et al., 1998; Poshakwale, 2002; and Liow and Webb, 2008). Despite the fact that the BDS test is a very useful test in detecting a dependency in a time series, it does not reveal whether the dependence is due to the linear, non-linear structures, or chaos.

The BDS test is based on a simple principle. If a time series is generated by an identically independently distributed (IID), the probability that the distance between any pair of observation is smaller than an arbitrary number should be the same for all pairs.

$$
p\left(\left|X_{t}-X_{t+k}\right|<\varepsilon\right)=p\left(\left|X_{t}-X_{t+l}\right|<\varepsilon\right)=p_{1}(\varepsilon)
$$

where $k$ and $l$ are arbitrary numbers. Therefore, for any IID financial time series, the joint probabilities that each pair of a sample will satisfy the condition (the distance is less than epsilon) can be expressed as:

$$
\prod_{d=1}^{m} p\left(\left|X_{t+d}-X_{t+k+d}\right|<\varepsilon\right)=p_{m}(\varepsilon)=p_{1}{ }^{m}(\varepsilon)
$$

The BDS test statistics is then calculated based on the above equality condition as:

$$
W_{m}(l)=\frac{\sqrt{T}\left[p_{m}(\varepsilon)-p_{1}^{m}(\varepsilon)\right]}{\sigma_{m}(\varepsilon)}
$$

where $m$ is embedding dimension and it is common to report the BDS test statistics on the first 6 embedding dimensions. Epsilon is chosen based on some features of the sample such as the standard deviation of the sample.

We performed the BDS test on each series of the ISE 100 index, Turkish/\$ exchange rate, Turkish inflation, and interbank overnight rate series. The results are reported in Table 2 . The null hypothesis of no dependence is rejected for all four series for the whole sample. We further fit different linear autoregressive moving (ARIMA) models to determine whether dependence is caused by linear structures inherent in the series. Unfortunately, there is no ARIMA model that explains the variation in the monthly Istanbul 100 stock price index. For the Turkish/\$ exchange rates, the autoregressive model with a constant term and 11 lags is the best fitted model. The Turkish interbank overnight interest rates can be described as an autoregressive model with significance in lags 4 and 7. Lastly, the best characterization of the inflation 
series is the autoregressive and moving average at lag 12 . The residuals obtained from these fitted models are then subjected to the BDS test. However, the BDS test results do not change, suggesting that the use of autoregressive moving model does not help to remove any potential linear dependence ${ }^{3}$. In summary, dependence found in all series is not caused by linear structures ${ }^{4}$. We further investigate the presence of non-linear and chaos as a possible explanation of dependence in the ISE 100 index returns. The nonlinear Generalized Autoregressive Conditional Heteroscedasticity (GARCH) model was fitted to the data prior to testing in order to filter out conditional heteroscedasticity. The best fitting model appears to be a model with one significant GARCH term and one threshold order based on the Bayesian Information Criterion. The BDS test statistics are then calculated on the residuals from the fitted GARCH model.

Table 2. BDS Test Statistics on the Residuals from the GARCH Fitted Models The ISE 100 Index, Turkish Lira/\$ Exchange Rate, Interbank Overnight Rate, and Inflation

\begin{tabular}{ccccc}
\hline $\begin{array}{c}\text { Dimension } \\
(\mathrm{m})\end{array}$ & $\begin{array}{c}\text { ISE 100 } \\
\text { Return }\end{array}$ & $\begin{array}{c}\text { Turkish Lira/\$ } \\
\text { Exchange Rate }\end{array}$ & $\begin{array}{c}\text { Interbank Overnight } \\
\text { Rate }\end{array}$ & $\begin{array}{c}\text { Consumer Price } \\
\text { Index }\end{array}$ \\
\hline & & & & \\
2 & 0,0012 & $0.0390^{* * *}$ & $0.0804^{* * *}$ & $0.0259^{* * *}$ \\
3 & 0,0047 & $0.1018^{* * *}$ & $0.1636^{* * *}$ & $0.0466^{* * *}$ \\
4 & $0.0156^{*}$ & $0.1576^{* * *}$ & $0.2317^{* * *}$ & $0.0658^{* * *}$ \\
5 & $0.0260^{* * *}$ & $0.1979^{* * *}$ & $0.2755^{* * *}$ & $0.0780^{* * *}$ \\
6 & $0.0334^{* * *}$ & $0.2226^{* * *}$ & $0.3057^{* * *}$ & $0.0877^{* * *}$
\end{tabular}

\begin{abstract}
Notes: The monthly ISE 100 returns are continuously compounded. The numbers in each column represent the BDS test statisics. The study period for ISE 100 index, Turkish Lira/\$ exchange rate, and consumer price index is from January 1988 to July 2013. The study period of Interbank overnigh rate from Turkey is from January 1990 to July 2013. The BDS test statistics are conducted on the raw data of each variables. The embedding dimensions $(\mathrm{m})$ are from 2 to 6 . The bootstrapped p-value for the BDS test statistics are calculated and used because of the samll samples. ${ }^{*}$ Indicates significance at the $10 \%$ level, ${ }^{* *}$ Indicates significance at the $5 \%$ level, and ${ }^{* * *}$ Indicates significance at the $1 \%$ level.
\end{abstract}

We found similar results when the BDS test is applied to the residuals from the fitted GARCH models. Table 2 shows the estimated values of the BDS test on the GARCH residuals from each series for embedding dimensions from 2 to 6 . The null hypothesis of IID in residuals is unequivocally rejected in every case of the three macroeconomic variables and at the higher dimensions ( $m=4,5$ and 6$)$ of the Turkish stock market index. Therefore, non-linear autoregressive models did not help to explain the dependence in the series. The inflation rate, exchange rate, and interest rates significantly explain the variance in log index returns. However, these important macroeconomic variables did not help to explain the nonlinear dependence in index returns. In other words, non-linear dependence in stock index returns cannot be attributed to these key macroeconomic variables.

The findings of non-IID in both linear ARMA and nonlinear GARCH filtered series virtually suggest that the Turkish stock market index and its corresponding macro-economic variables might possibly be generated by a nonlinear model. Our results endorse the findings of nonlinear dependence in financial markets reported by many prior studies including Liow and Webb (2008) and others.

When the sample is divided into a pre- and post-May 2000 periods, the dependency persists in both periods. However, the null hypothesis of independence is rejected more strongly at the traditional significant level in the post-May 2000 sample period compared to the pre-May 2000 sample $^{5}$. 


\subsection{K-map and Z-map Analysis}

It is undeniable that the BDS test is a powerful test in detecting a dependency in a time series. However, it does not reveal whether the dependence is in the form of linear, non-linear, or chaos structures. The BDS test will therefore reject IID if the time series is chaotic. According to Heish (1991), a chaotic time series can be generated by nonlinear deterministic process which may look random, but not all non-linear dynamics exhibit a chaotic behavior.

The K-map and Z-map analysis is further implemented to determine whether the dependency in the ISE series is driven by a chaotic structure. Following the study by Larrain (1991), non-linear components in the ISE returns are modelled as K-map to represent chaotic behaviors. The linear behavior components in the log returns of ISE 100 are modelled as Z-map to include four important macro-economic factors such as inflation (CPI), interest rate (INT), gross domestic products (GDP), and Turkish/\$ currency value (EXRATE).

There is an increasing amount of empircial evidence points to the conclusion that stock market behvior can be predicted by a wide range of macro-economic factors (Balver et al., 1990; Breen et al., 1990; Campbell and Hamao, 1992; Pesaran and Timmerman, 1995). The inflation, gross domestic product, interest rate, and Turkish lia/\$ exchange rates are therefore included in the K-map and Z-map model to examine whether the dependence in the ISE 100 return can be explained by the movements of these macroeconomic variables or by the chaos process. Prior studies examining the predictability of the conditional volatility of macro variables on equity volatility include Schwert (1989), Liljeblom and Stenius (1997), and Morelli (2002). Schwert (1989) reports weak evidence of the predictability of the conditional volatility of inflation, industrial production, and money supply on the volatilities of the U.S. stock market. Liljeblom and Stenius (1997), on the other hand, found that between one-sixth and two-thirds of the variations in the Finnish stock market could be explained by changes in the volatility of the same three macroeconomic variables plus terms of trade. Morelli (2002) finds no explanatory power for the volatility of the macroeconomic variables of exchange rate, industrial production, inflation, real retail sales, and money supply on the volatility of the U.K. stock markets. In addition, previous studies establish that these variables can be used as representative macro variables with explanatory power for the movement of stock and REIT returns (Jaffe and Mandelker, 1976; Chen and Tzang (1988); Lu and So, 2001). Jaffe and Mandelker (1976) document a negative relationship between inflation and securities returns. Chen and Tzang (1988) present evidence that changes in the federal fund rate provide long-term information about the movement of securities returns. Lu and So (2001) find that changes in real economic activity and risk premium are positively correlated with returns on commons stocks. As there is substantial evidence on the significant role of these macroeconomic variables in explaining the security return, it is legitimate to include these variables in the structural Z-map model.

The K-map and Z-map of the ISE returns can be shown as follows:

$R_{t}=c+\beta_{1} R_{t-1}^{1}+\beta_{2} R_{t-1}^{2}+\beta_{3} R_{t-1}^{3}+\beta_{4} R_{t-1}^{4}+\beta_{5}($ EXRATE $)+$

$\beta_{6}(C P I)+\beta_{7}(I N T)+\beta_{8}(G D P)+\varepsilon_{t}$

If the estimated coefficients on the k-map variables (c, $\beta_{1}, \beta_{2}, \beta_{3}$, and $\beta_{4}$ ) are more significant and powerful than the estimated coefficients of the Z-map variables $\left(\beta_{5}, \beta_{6}, \beta_{7}\right.$, and $\beta_{8}$ ), it indicates that the dynamic behavior of the ISE return is driven by erratic and chaotic behavior.

We first estimated the model with a stepwise regression to determine the best degree of nonlinearity used in the K-map model. Once the fitted model is found, the coefficients are estimated via ordinary least square regression (OLS). Lastly, the OLS is performed on the full model that is composed of significant non-linear K-map components and linear fundamental economic Z-map components. The results of the K-map and Z-map analysis on the ISE returns are presented in Table 3. Only three variables are included in the model, which are the change in Turkish/\$ exchange rates, de-trended Turkish interest rates, and inflation rates. All three fundamental economic variables coefficients are significant. The estimated 
coefficients of Turkish Lira value and inflation rates are negative, but the coefficient estimate of interest rate is positive. This is not very surprising. When the economy is stronger, the stock market performs well, Turkish Lira becomes stronger in relative to U.S. dollars and interest rates tend to be low. The opposite usually holds true when the future outlook for the economy is weak. Surprisingly, none of the K-map nonlinear components is significant. Although these results suggest that non-linearity in the ISE returns as reported by the BDS is not consistent with the chaos behavior, it does not rule out the possibility of a chaos. There are many specific tests for chaos that should be further implemented.

Table 3. Summary of OLS Regressions of ISE 100 Returns and Macro-Economic Variables: The K-map and Z-map (January 1988 to July 2013)

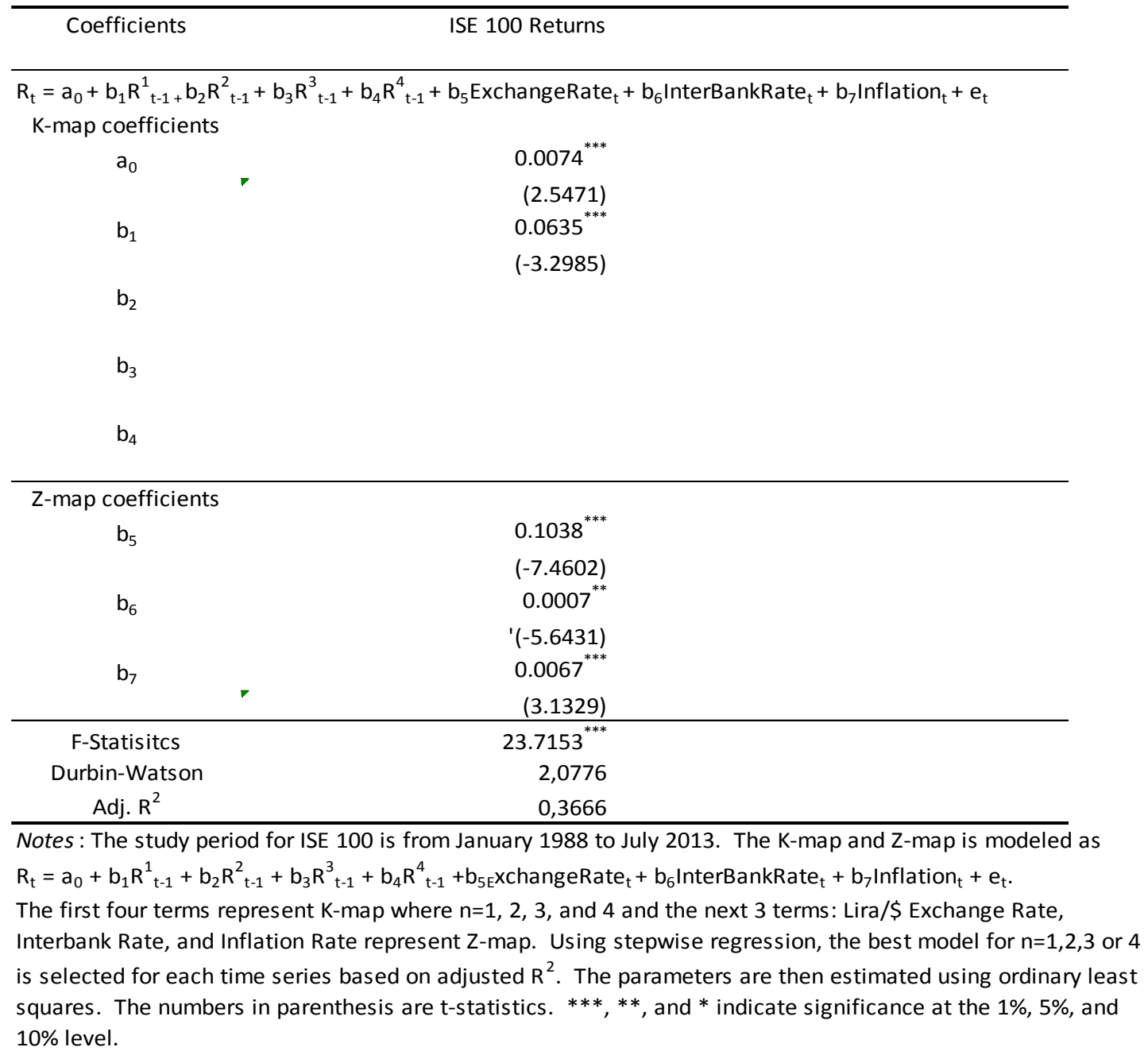

When the K-map and Z-map analysis is performed on the two sub-periods in accordance with the structural break in May 2000, the results do not change. None of the K-map coefficients are significant, suggesting the lack of chaos behavior in the ISE return during the pre-May 2000. However, in the post-May 2000 sample periods, $\beta_{1}$ and $\beta_{6}$ become significant. That is, the first lag of log returns and inflation coefficients become significant ${ }^{6}$.

\subsection{Markov Chain Test}

The second order Markov chain test developed by McQueen and Thorley (1991) is implemented to statistically detect nonlinearity in the ISE 100 index. The advantage of the Markov chain test is that it can reveal non-linear dependence without requiring series under investigation to be normally distributed. Moreover, the dependence is tested based on what happened in the prior two periods. This form of 
dependency conditioning on the most two recent periods is very crucial from the perspective of efficient markets hypothesis. The null hypothesis based on the Markov chain test is that the behavior of financial time series follows a random walk. More specifically, the Markov chain technique tests whether the probability of observing a positive or negative return in a given period is the same, regardless of whether the returns in previous two periods are negative or positive. This testing procedure starts with determining the maximum likelihood estimates of four transition probabilities $\left(\lambda_{i, j}\right)$. The transition probabilities can be defined as a probability of observing a positive or negative return in the current period given the four different possible paths in last two periods: positive-positive, positive-negative, negative-positive, and negative-negative returns. The log likelihood ratio test is then calculated based on unrestricted case and restricted case specified in each of the tested null hypotheses ${ }^{7}$.

A two-state second-order Markov process, $I_{t}$, can be defined as follows:

$I_{t}=\left\{\begin{array}{l}1, R_{t} \geq 0 \\ 0, R_{t}<0\end{array} \quad\right.$ where $R_{\mathrm{t}}$ is the monthly return at time $t$

To test the randomness of each security returns, a two-order transition probability $\left(\lambda_{\mathrm{ij}}\right)$ is constructed based on the behavior of process $\left\{I_{t}\right\}$ :

$\lambda_{i j}=\operatorname{Prob}\left(I_{t}=0 \mid I_{t-2}=i, I_{t-1}=j\right)$

where $i$ and $j$ can take the value of either 1 or $0 . \lambda_{00}$ is the probability that a negative return will continue to persist in the current period given two preceding negative returns. Likewise, $\left(1-\lambda_{00}\right)$ is the probability that a sequence of two negative returns will revert to a positive return in the current period.

The random walk hypothesis posits that the probability of either state $0\left(\right.$ i.e., $\left.I_{t}=0\right)$ or state $1\left(I_{t}=1\right)$ should be invariant to any prior two-state sequence. A rejection of the null hypothesis, therefore, indicates the presence of nonlinear dependence in the return dynamics. The following seven null hypotheses are tested:

1. $H_{1, \text { null }}: \lambda_{00}=\lambda_{01}$ versus $H_{1, \text { alt }}: \lambda_{00} \neq \lambda_{01}$

2. $H_{2, \text { null }}: \lambda_{00}=\lambda_{10}$ versus $H_{2, \text { alt }}: \lambda_{00} \neq \lambda_{10}$

3. $H_{3, \text { null }}: \lambda_{00}=\lambda_{11}$ versus $H_{3, \text { alt }}: \lambda_{00} \neq \lambda_{11}$

4. $H_{4, \text { null }}: \lambda_{01}=\lambda_{10}$ versus $H_{4, \text { alt }}: \lambda_{01} \neq \lambda_{10}$

5. $H_{5, \text { null }}: \lambda_{01}=\lambda_{11}$ versus $H_{5, \text { alt }}: \lambda_{01} \neq \lambda_{11}$

6. $H_{6, \text { null }}: \lambda_{10}=\lambda_{11}$ versus $H_{6, \text { alt }}: \lambda_{10} \neq \lambda_{11}$

7. $H_{7, \text { null }}: \lambda_{00}=\lambda_{01}=\lambda_{10}=\lambda_{11}$ versus $H_{7, \text { alt }}: \lambda_{00} \neq \lambda_{01} \neq \lambda_{10} \neq \lambda_{11}$ 
Table 4. Markov Chain Tests for ISE 100 Returns (January 1988 to July 2013)

\begin{tabular}{lcccc}
\hline & & \multicolumn{3}{c}{ ISE 100 Return } \\
\hline & \multicolumn{3}{c}{ Current State } \\
0 & Previous State & 0 & 1 \\
0 & 0 & 17 & 34 \\
0 & 1 & 32 & 41 \\
1 & 0 & 34 & 39 \\
1 & 1 & 41 & 65 \\
\hline
\end{tabular}

\section{MLE Estimates}

$\begin{array}{cc}I_{00} & 0,3333 \\ s\left(I_{00}\right) & (0.0660) \\ I_{01} & 0,4384 \\ s\left(I_{01}\right) & (0.0581) \\ I_{10} & 0,4657 \\ s\left(I_{10}\right) & (0.0584) \\ I_{11} & 0,3868 \\ s\left(I_{11}\right) & (0.0473)\end{array}$

Likelihood Ratio Hypothesis Test

$\begin{array}{cc}\mathrm{H}_{1}: I_{00}=I_{01}(\text { LRT }) & 1,3967 \\ \text { P-value } & (0.2373) \\ \mathrm{H}_{2}: I_{00}=I_{10}(\text { LRT }) & 2,1953 \\ \text { P-value } & (0.1384) \\ \mathrm{H}_{3}: I_{00}=I_{11}(\text { LRT }) & 0,4258 \\ \mathrm{P} \text {-value } & (0.5141) \\ \mathrm{H}_{4}: I_{01}=I_{10}(\text { LRT }) & 0,1106 \\ \mathrm{P} \text {-value } & (0.7394) \\ \mathrm{H}_{5}: I_{01}=I_{11}(\text { LRT }) & 0,4750 \\ \mathrm{H}_{6}: I_{10}=I_{11}(\text { LRT }) & (0.4907) \\ \mathrm{P} \text {-value } & 1,1050 \\ \mathrm{H}_{7}: I_{00}=I_{01}=I_{10}=I_{11} & (0.2932) \\ P \text {-value } & 2,6740\end{array}$

Notes: ISE 100 returns are continuously compounded. The study period for ISE 100 returns is from The study period for all types of REITs is from January 1988 to July 2013. The number in parenthesis below the Maximum Likilihood estimates (MLE) of the transition probabilities $\mathrm{I}_{\mathrm{ij}}$ are the associated asymptotic standard errors, or $s\left(I_{i j}\right)$. The critical values for $H_{1}$ to $H_{6}$ are $c^{2}(1)=6.63$ at the $1 \%$ level, $c^{2}(1)=3.84$ at the $5 \%$ level, and $c^{2}(1)=2.71$ at the $10 \%$ level. The critical values for $\mathrm{H}_{7}$ is $c^{2}(3)=11.3$ at the $1 \%$ level, $c^{2}(3)=7.82$ at the $5 \%$ level, and $c^{2}(3)=6.25$ at the $10 \%$ level. P-Value is the marginal significance level, which is the probability of obtaining that value of the LRT or higher under the null hypothesis. ${ }^{* *}, * *$, and $*$ denote significance at the $1 \%, 5 \%$, and $10 \%$ level, respectively.

Table 4 reports the result of second-order Markov chain test on the ISE 100 returns. The unconstrained estimate of $\hat{\lambda}_{00}(0.3333)$ is smaller than $\left(1-\hat{\lambda}_{00}\right)$, which suggests that a positive return is more likely to follow two prior negative returns. The mean reversion is not the case when the two prior returns are positive. The unconstrained estimate of $\hat{\lambda}_{11}(0.3868)$ is smaller than $\left(1-\hat{\lambda}_{11}\right)$, suggests a presence of the persistence of positive returns. These transition probabilities also point to the existence of predictable components in the ISE 100 returns where positive return is more likely to occur after two consecutive sequence of either negative or positive returns. 
The log likelihood ratio test is further conducted to formally test for the random walk hypothesis in the ISE 100 returns. The test is performed by re-estimating $\hat{\lambda}_{00}$ and $\hat{\lambda}_{11}$ based on the restriction imposed in each of the seven null hypotheses. Under the less restrictive null hypotheses 1 to $6\left(H_{1}\right.$ to $\left.H_{6}\right)$, the probability of observing negative returns in the current period should be the same regardless of what happened in the prior two periods. For more restrictive null hypothesis $7\left(\mathrm{H}_{7}\right)$, the probability of negative or positive occurrence in the current period should be same regardless of pattern in prior two periods. The log likelihood ratio tests fail to reject any of these hypotheses of randomness. That is, there is no evidence of non-linear dependency in the monthly logarithmic ISE index returns. The sample data is divided into two parts to investigate the effect of a possible structural break on the results. When the Markov chain tests are repeated for the two sub-sample periods, the results were the same as the full period. The null hypothesis of randomness cannot be rejected in each of seven hypotheses at the traditional significant level ${ }^{8}$. The finding here suggests that there is no short term (3-months) dependency in the ISE index returns. Therefore, the dependence detected by the BDS test must be a long-term in nature.

\subsection{Time Reversibility Test}

The non-linear time reversibility test (TR) is formally conducted to test for a non-linear dependence or an asymmetric pattern in a time series. The test is first implemented by Ramsey and Rothman (1996). The time reversibility is applied to further characterize the return dynamics of the ISE 100 . For a time series that exhibits symmetric or time reversible patterns, reversing the time axis will not change the behaviors of the time reversible series. Therefore, a rejection of time reversibility in the ISE 100 return indicates the presence of asymmetric patterns consistent with the non-random walk behaviors.

According to Ramsey and Rothman (1996), a time series $\left\{X_{t}\right\}$ is time reversible if $E\left[X_{t}^{i} * X_{t-k}^{j}\right]=$ $E\left[X_{t}^{j} * X_{t-k}^{i}\right]$ for all $i, j, k \in N$, and $k$ is the lag periods. For $i=1$ and $j=2$, a bicovariance function to test for the time reversibility can be defined as:

$$
\gamma_{2,1}(k)=\left\{E\left[X_{t}^{2} * X_{t-k}\right]-E\left[X_{t} * X_{t-k}^{2}\right]\right\}
$$

The sample estimate of this bicovariance function can be calculated with the following formula

$$
\hat{\gamma}_{2,1}(k)=\frac{\sum_{t=k+1}^{T} X_{t}^{2} * X_{t-k}-\sum_{t=k+1}^{T} X_{t} * X_{t-k}^{2}}{T-k}
$$

Under the null hypothesis that $\left\{X_{t}\right\}$ is time reversible, the expected value of $\hat{\gamma}_{2,1}(k)$ is zero for all lags $k$. The standardized TR test statistic, $\frac{\hat{\gamma}_{2.1}(k)}{\operatorname{VAR}\left[\hat{\gamma}_{2.1}(k)\right]^{1 / 2}}$, which is the ratio of sample estimate of bicovariance function to its standard deviation is calculated. The Monte Carlo simulation is used to generate the critical values for the standardized TR test statistics. An ARIMA model is first estimated for the log of ISE returns. Based upon the fitted ARMA model, a Monte Carlo simulation is conducted to generate 1,000 values of $\widehat{\gamma}_{2,1}(k)$ and its corresponding standard deviations for each lag $k$. The standardized time reversibility statistics are reported in Table 5 for the first 10 lags. 
Table 5. Time Reversibility Test on Monthly Returns of ISE 100 (January 1988 to July 2013)

Lag K ISE 100 Return $\quad$ Residuals from ARMA Model

\begin{tabular}{|c|c|c|}
\hline$k=1$ & 0,6749 & 0,4934 \\
\hline$k=2$ & $-1,1310$ & $-0,8069$ \\
\hline$k=3$ & $2.6746^{* * *}$ & $2.0068^{* *}$ \\
\hline$k=4$ & $-0,2397$ & $-0,1686$ \\
\hline$k=5$ & 0,0490 & 0,0533 \\
\hline$k=6$ & $-1.8068^{*}$ & -1.3048 \\
\hline$k=7$ & $-0,5080$ & $-0,3639$ \\
\hline$k=8$ & 0,4917 & 0,3713 \\
\hline$k=9$ & 0,2474 & 0,2056 \\
\hline$k=10$ & $2.3539^{* *}$ & $1.7138^{*}$ \\
\hline$P_{1,10}$ & $18.31^{* *}$ & 9,9100 \\
\hline Rejection Type & \multicolumn{2}{|c|}{ Type II: Time Irreversibility is due to Linear but NonGaussian Innovations } \\
\hline \multicolumn{3}{|c|}{$\begin{array}{l}\text { Notes: Monthly ISE } 100 \text { returns are continuously compounded. Numbers in the first column is the } \\
\text { standardized time reversibility test statistics of real returns on ISE } 100 \text {. Standardized time reversibility } \\
\text { test statistics are calculated as } g_{21}(\mathrm{k}) /\left[\operatorname{var}\left(\mathrm{g}_{21}(\mathrm{k})\right)\right]^{1 / 2 .} \text { The } \operatorname{var}\left(\mathrm{g}_{21}(\mathrm{k})\right) \text { is estimated via Monte Carlo simulation } \\
\text { when the TR test is performed on the real returns. The next column reports the standardized TR test on the } \\
\text { ARMA residuals on the ISE } 100 \text { returns. The significance of the TR test statistics for each lag is determined } \\
\text { via the distribution generated through Monte Carlo simulation. } \mathrm{P}_{1,10} \text { is the time reversibility portmantaeu } \\
\text { statistics calculated using equation }(9), \text { which provides a joint test on a set of standardized TR test statistics } \\
\text { value. With } 10 \text { degrees of freedom, } \mathrm{c}_{0.9}^{2}=15.99, \mathrm{c}_{0.95}^{2}=18.30, \mathrm{c}_{0.99}^{2}=23.20 \text {. Type I rejection is the time } \\
\text { irreversibility due to non-linear but Gaussian innovations. Type II rejection is the time reversibility due to } \\
\text { linear but non-Gaussian innoviations. Time reversibility indicates symmetry. }{ }^{* * *}, * *, * \text { indicates significance } \\
\text { at the } 1 \%, 5 \% \text {, and the } 10 \% \text { level. }\end{array}$} \\
\hline
\end{tabular}

To jointly test for the time reversibility for several lags of $k$, the time reversibility portmanteau statistic is calculated as:

$$
P_{m, n}=\sum_{k=m}^{n}\left(\frac{\widehat{\gamma}_{2,1}(k)}{\widehat{\sigma}_{\gamma}}\right)^{2}
$$

This portmanteau statistic is distributed as $\chi^{2}$ with $n-m+1$ degrees of freedom.

Once the time irreversibility or asymmetry is established, we can further identify whether asymmetry is due to the functional forms or the asymmetric innovation of data generating process. According to Ramsey and Rothman (1996), the sources of time irreversibility can be in two forms. First, irreversibility in time series is caused by non-linear functional form with Gaussian innovations (Type I time irreversibility). Second, irreversibility in time series is also caused by linear functional form with nonGaussian innovations (Type II time irreversibility). These two types of time irreversibility can be differentiated by calculating standardized TR test statistics on the ARMA residuals. The $\operatorname{VAR}\left[\widehat{\gamma}_{2,1}(k)\right]^{1 / 2}$ is calculated $a^{9}$ :

$$
\operatorname{var}\left[\hat{\gamma}_{2,1}(k)\right]^{1 / 2}=2\left(\mu_{4} \mu_{2}-\mu_{3}^{2}\right) /(T-k)-2 \mu_{2}^{3}(T-2 k) /(T-k)^{2}
$$

Where $\mu_{3}=E\left[X_{t}^{2}\right], \mu_{3}=E\left[X_{t}^{3}\right]$, and $\mu_{4}=E\left[X_{t}^{4}\right]$. When the null hypothesis of time reversibility is rejected under both the raw ISE returns and ARMA residuals, we can conclude that the asymmetric behavior is caused by the non-linearity in the functional form of the model. On the other hand, 
if the null hypothesis of time reversibility is rejected only for the raw return, but not the ARMA residuals, one can conclude that asymmetric behavior is caused by the non-Gaussian innovations.

Table 5 reports the results of the TR test for lags 1 to 10 and its joint test of time reversibility for all lags for the ISE 100 returns and the ARMA residuals. The null hypothesis of time reversibility in the ISE 100 return is rejected for lags 3,6 , and 10 at the traditional significant level. Similar finding is reported when the TR test is applied on the ARMA residuals. The null hypothesis of time reversibility is also rejected at lags 3 and 10 at the traditional significant level. The portmanteau statistics, $P_{1,10}$, for joint test of time reversibility of 10 lags indicate a strong rejection of the time reversibility in the ISE 100 returns at the 5\% significant level. However, when the same portmanteau statistics are calculated on the ARMA residuals, the results suggest that the residuals are time reversible. The finding here suggests that the log returns of Istanbul Stock Exchange (ISE) 100 index are asymmetric or time irreversible. The asymmetric behavior is not caused by the nonlinearity in the ISE returns model, but by the non-Gaussian innovations of the return generating process. When the sample is divided according to the structural break in May 2000, the TR portmanteau statistics report the same results in both periods. That is, the ISE 100 index can be modeled with a linear function with non-Gaussian error terms. In other words, the innovations in the ISE 100 series are asymmetric, causing an asymmetric behavior in the ISE equity returns.

\subsection{Duration Dependence Test}

The last technique implemented here is to examine whether non-linear behavior in the ISE 100 return can be explained by a financial bubble. For this purpose, a duration dependence test developed by McQueen and Thorley (1994) is employed. The Duration dependence test is based on a logit regression to define the relationship between the run length and the probability of crash ${ }^{10}$. The presence of a rational bubble would suggest that the probability of obtaining a negative return (a bubble bursts) should decrease as the length of positive return increases (a bubble grows). In other words, the hazard rate should be negative.

The duration dependence test starts with dividing the returns into a group of positive and negative returns. The numbers of positive and negative runs at a particular length $k$ are then counted. A run is a sequence of abnormal returns with the same sign. Suppose $X_{t}$ is a time series with $n$ abnormal returns, a positive run with length $k$, or $R_{k}$, can be defined as:

$$
R_{k} \in\left\{X_{i}<0 \mid X_{i-1}>0, \ldots, X_{i-k}>0, X_{i-k-1}<0\right\} \forall i \leq n \text { and } k \leq i
$$

From the perspective of a long investor, the hazard $\left(h_{i}\right)$ is the possibility of the bubble may burst at any given time period. For a rational bubble to survive, the hazard rate should decline as the length of the positive run increases. Therefore, the hazard rate $\left(h_{i}\right)$ can be expressed as a function of log of the lag length.

$$
h_{i}=\frac{1}{1+e^{-d_{i}}}
$$

where, $h_{i}$ is the probability that the run will end, and

$$
d_{i}=\alpha+\beta \operatorname{Ln} i
$$

$d_{i}$ is the likelihood of the run will end, and $i$ is the lag length. The parameters are estimated via the logit regression model. Once the parameters are obtained, a likelihood ratio test is carried out to test whether restricting the beta to zero significantly decreases the log likelihood.

The results of the duration dependence test are presented in Table 6. During the sample period studied, there were a total of 146 runs (73 positive runs and 73 negative runs). The longest run is a 9month positive run. In a rational expectations bubble, the hazard rate of a possibility that the bubble might burst should decrease as the duration of the bubble of positive runs gets longer. According to our results, 
the sample hazard rates of the ISE 100 do not decrease monotonically. Although the relationship between the duration of the run and the probability of a run to end is negative with $\beta=-0.0833$, it is not statistically significant. The log likelihood ratio test fails to reject the null hypothesis of no bubble $(\beta=0)$. These results suggest that there is no strong statistical evidence that supports the hypothesis that there is a rational bubble in the ISE 100 index returns. However, there is a statistically significant relationship between a run length and a sample hazard rate in negative runs. The sample hazard rate monotonically increases with the duration length. The null hypothesis of $\beta=0$ is rejected in favor of $\beta=3.1907$ at the $10 \%$ significance level. This suggests that the probability of a negative run ends does increase as the run gets longer, which is consistent with a mean reversion process.

Table 6. Sample Hazard Rate and the Duration Dependenct Test on Monthly Returns of ISE 100 (January 1988 to July 2013)

\begin{tabular}{|c|c|c|c|c|c|c|}
\hline & \multicolumn{3}{|c|}{ Positive Runs } & \multicolumn{3}{|c|}{ Negative Runs } \\
\hline \multirow{4}{*}{$\begin{array}{c}\text { Run } \\
\text { Length }\end{array}$} & \multicolumn{2}{|l|}{ Actual } & Sample & \multicolumn{2}{|l|}{ Actual } & Sample \\
\hline & \multicolumn{2}{|l|}{ Run } & Hazard & \multicolumn{2}{|l|}{ Run } & Hazard \\
\hline & \multicolumn{2}{|l|}{ Counts } & Rate $^{3}$ & \multicolumn{2}{|l|}{ Counts } & Rate \\
\hline & \multicolumn{3}{|c|}{ Total Positive Runs $=73$} & \multicolumn{3}{|c|}{ Total Negative Runs $=73$} \\
\hline 1 & 32 & & 0,4384 & 38 & & 0,5205 \\
\hline 2 & 16 & . & 0,3902 & 24 & - & 0,6857 \\
\hline 3 & 10 & 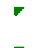 & 0,4000 & 7 & & 0,6364 \\
\hline 4 & 5 & . & 0,3333 & 3 & & 0,7500 \\
\hline 5 & 4 & & 0,4000 & 1 & 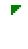 & 1,0000 \\
\hline 6 & 1 & . & 0,1667 & & & \\
\hline 7 & 2 & & 0,4000 & & & \\
\hline 8 & 2 & & 0,6667 & & & \\
\hline 9 & 1 & & 1,0000 & & & \\
\hline
\end{tabular}

\begin{tabular}{ccc}
\hline Total Positive and Negative Runs & 146 & \\
& & \\
\hline Log-Logistic Test & & 0,0787 \\
a & $-0,1687$ & $(0.5529)$ \\
(t-value) & $(-1.2547)$ & $0.4596^{*}$ \\
b & $-0,0833$ & $(1.7634)$ \\
(t-value) & $(-0.5823)$ & $3.1097^{*}$ \\
LRT $^{4}$ of $\mathrm{H}_{1}: \mathrm{b}=0$ & 0,3398 & $(0.0778)$ \\
(p-value) & $(0.5599)$ &
\end{tabular}

Notes: Duration Test is performed on the monthly real return of ISE 100. Actual run counts do not include the partial runs which may occur at the beginning or at the end of the period investigated. Total runs are the numbers of total positive and negative runs. The sample hazard rate, $h_{i}=N_{i} /\left(M_{i}+N_{i}\right)$, indicates probability that a run ends at length $i$ provided that is lasts until $i$. The likelihood ratio test (LRT) of the null hypothesis of no duration dependence of constant hazard rate $\left(\mathrm{H}_{1}: \mathrm{b}=0\right)$ is asymptotically distributed $\mathrm{c}^{2}$ with one degree of freedom. The critical values are 3.841 and 2.706 at $5 \%$ and $10 \%$ significance level. P-value is the marginal significance level--the probability of obtaining the calculated value of LRT or higher under the null hypothesis.

$* * *$ Indicates significance at the $1 \%$ level, ** indicates significance at the $5 \%$ level, and * Indicates significance at the $10 \%$ level.

When the duration dependence is applied on the period before May 2000, there is no significant difference in the results from the full period. However, the significant relationship between negative runs and the run length disappears when the duration dependence test is applied to the post-May 2000 period. 


\section{Conclusions}

The dynamics of the Istanbul Stock Exchange (ISE) 100 index is explored in this study using the data during the last 25 years. The main motivation of this paper is to determine the source and nature of any dependency that remains after the series made stationary. This paper applies a battery of tests on the ISE 100 index to uncover its non-linear dynamics. The ISE 100 index is nonstationary but the log returns of the index are stationary. There is evidence of the dependency in the log returns of Turkish equity market index. Based on the BDS test, the dependency is not in a linear form since no ARIMA model helps remove any dependency. Moreover, the dependence cannot be explained by nonlinear autoregressive process (GARCH). Further K-map and Z-map analysis also suggests that nonlinear dependence is not caused by erratic and chaotic dynamics and it is not driven by important macro-variables.

The Markov chain test results suggest that the persistent dependence is not short term (3-months or less) in nature. The log ISE 100 return series are time irreversible or asymmetric. However, the ARIMA residuals are time reversible. This suggests that asymmetric nonlinearity in the ISE 100 index is caused by non-Gaussian innovations. The duration dependence test suggests that there is no evidence of rational bubble in the log ISE 100 returns. There is some evidence that there might be a structural break that occurred in May 2000. However, when all the tests are applied on the data before and after May 2000, the results do not vary significantly.

These results suggest that overall Turkish equity market is relatively efficient. The predictable component of the ISE 100 index is nonlinear, but non-chaotic and bubble-free. The dependency in the return is uncorrelated with the movement of any macro-economic factors and cannot be explained by conditional autoregressive variance. Future studies should compare the forecasting performances of the ISE 100 index based on different non-linear models.

The finding of this paper provides some important implications for practitioners, investors, policymakers, and regulators. Based on the comprehensive analysis of the dynamics in the Turkish equity, the Turkish equity market can be viewed as a relatively efficient market. The market participants should be able to trust the market forces to obtain a fair return for their investments in the ISE 100 index. Therefore, practitioners can still rely on the fundamental valuation methods as a tool for making successful investment decision and enhancing portfolio performance. The overwhelming finding of linear dynamic behavior in the ISE 100 index suggests that investor can use linear model to accurately forecast the securities price of the equities in Turkey. The lack of sufficient evidence in our finding that points to the presence of speculative bubbles in Turkey further substantiates the notion of efficiency in Turkey markets. However, policymakers should continue to be vigilant in keeping the market free from manipulation and other adverse influences particularly from the large players in order to protect small investors. Regulators should have policy in place such as safety net and circuit breakers implementation to prevent instability and sudden market crashes. Regulators should also continue to closely regulate the Turkish equity market and effectively monitor the investment practices of market participants in order to maintain and enhance the efficiency of the equity market in Turkey.

\section{End Notes}

${ }^{1}$ Raw return series is not used since it is not stationary.

${ }^{2}$ To provide the reliability of the unit root tests results, we have implemented several other unit root tests. These unit root tests are Elliot-Rothenberg-Stock (1996), Kwiatkowski et al (1992), Schmidt-Phillips (1992), Zivot-Andrews (1992), Lee-Strazicich (2003), and Lumsdaine-Papell (1997) tests. Most of these tests allow for structural breaks in the data. We implemented several alternatives of structural breaks in trend, intercept, and both trend and intercept. In addition, we carried out the unit root test with 1,2, and 3 structural break alternatives. All the unit root tests provide consistent results of rejecting a unit root in the ISE 100 series.

${ }^{3}$ The statistics slightly change but all of them are still significant. That is, the results reject the null hypothesis of independence. 
${ }^{4}$ The BDS test results on the residuals from a linear ARMA model are not reported here but are available from the authors upon request.

${ }^{5}$ To conserve space, the results are not reported here but are available upon request.

${ }^{6}$ To conserve the space, the results of K-map and Z-map analysis for the two sub-periods are not reported here but a are available upon request from the authors.

${ }^{7}$ See McQueen and Thorley (1991) for more details.

${ }^{8}$ To conserve the space, the results of Markov chain test for the two sub-periods are not reported here.

${ }^{9}$ Ramsey and Rothman (1996) showed that the $\operatorname{VAR}\left[\widehat{\gamma}_{2,1}(k)\right]^{1 / 2}$ is calculated under the assumption that $\left[X_{t}\right]$ is a stationary sequence of IID random variables where $E\left[X_{t}\right]=0 \forall t$ and $E\left[X_{t}^{4}\right]<\infty$.

${ }^{10}$ For more details see McQueen and Thorley (1994).

\section{References}

Ammermann, P. A. and Patterson, D. M. (2003). The Cross-Sectional and Cross- Temporal Universality of Nonlinear Serial Dependencies: Evidence from World Stock Indices and the Taiwan Stock Exchange. Pacific-Basin Finance Journal, 11(2), 175-195.

Antoniou, A., Ergul, N., and Holmes, P. (1997). Market Efficiency, Thin Trading and Non-linear Behaviour: Evidence from an Emerging Market. European Financial Management, 3(2), 175-190.

Bali, T. G., Demirtas, K. O., and Levy, H. (2008). Nonlinear Mean Reversion in Stock Prices. Journal of Banking \& Finance, 32(5), 767-782.

Balvers, R.J., Cosimano, T.F., and McDonald, B. (1990) Predicting Stock Returns in an Efficient Market. Journal of Finance, 45, 1109-1128.

Bildirici, M., and Ersin, O. O. (2009). Improving Forecasts of GARCH Family Models with the Artificial Neural Networks: An Application to the Daily Returns in Istanbul Stock Exchange. Expert Systems with Applications, 36(4), 7355 7362.

Bollerslev, T. (1986). Generalized Autoregressive Conditional Heteroskedasticity. Journal of Econometrics, 31(3), 307327.

Bonilla, C. A., Romero-Meza, R., and Hinich, M. J. (2006). Episodic Nonlinearity in Latin American Stock Market Indices. Applied Economics Letters, 13(3), 195-199.

Boyacioglu, M. A. and Avci, D. (2010). An Adaptive Network-Based Fuzzy Inference System (ANFIS) for the Prediction of Stock Market Return: The Case of the Istanbul Stock Exchange. Expert Systems with Applications, 37(12), 7908-7912.

Breen, W., Glosten, L.R. and Jagannathan, R. (1990). Predicable Variations in Stock Index Returns. Journal of Finance, 44, 1177-1189.

Broock, W. A., Scheinkman, J. A., Dechert, W. D., and LeBaron, B. (1996). A Test for Independence Based on the Correlation Dimension. Econometric Reviews, 15(3), 197-235.

Campbell, J.Y. and Hamao, Y. (1992). Predictable Stock Returns in the United States and Japan: A Study of Long-Term Capital Integration. Journal of Finance, 47, 43-67.

Caraiani, P. (2014). What Drives the Nonlinearity of Time Series: A Frequency Perspective. Economics Letters. In press.

Chen, K.C. and Tzang, D (1988). Interest-Rate Sensitivity of Real Estate Investment Trusts. Journal of Real Estate Research, 3(3), 13-22.

Cutler, D. M., Poterba, J. M., and Summers, L. H. (1989). What Moves Stock Prices? Journal of Portfolio Management, 15(3), 4-12.

Elliott, G., T. Rothenberg and J. Stock (1996). Efficient Tests for an Autoregressive Unit Root. Econometrica 64 (4), 813-836.

Engle, R.F. (1982) Autoregressive Conditional Heteroskedasticity with Estimates of the Variance of United Kingdom Inflation. Econometrica, 50: 987-1007.

Erkus, H., \& Ugur, A. (2014). Nonlinear Market Behavior at the Istanbul Stock Exchange. Electronic Journal of Social Sciences, 13(49). 
Frank, M. Z. and T. Stengos. (1986) Measuring the Strangeness of Gold and Silver Rates of Returns. Discussion Paper No. 1986-13, Department of Economics, University of Guelph, 1989.

Hamilton, J. D. (1990). Analysis of Time Series Subject to Changes in Regime. Journal of Econometrics, 45(1), 39-70.

Harris, R. D. and Kucukozmen, C. C. (2001). Linear and Nonlinear Dependence in Turkish Equity Returns and Its Consequences for Financial Risk Management. European Journal of Operational Research, 134(3), 481-492.

Hinich, M. J. (1982). Testing for Gaussianity and Linearity of a Stationary Time Series. Journal of Time Series Analysis, 3(3), 169-176.

Hsieh, D. A. (1989). Testing for Nonlinear Dependence in Daily Foreign Exchange Rates. Journal of Business, 339-368.

Hsieh, D. A. (1991). Chaos and Nonlinear Dynamics: Application to Financial Markets. The Journal of finance, 46(5), 1839-1877.

Jaffe, J.F. and Mandelker, G. (1976). The Fisher Effect for Risky Assets: An Empirical Investigation. Journal of Finance, 3 (12), 447-458.

Kwiatowski, P. Phillips, Schmidt, P and Shin, Y.(1992). Testing the Null Hypothesis of Stationarity Against the Alternative of a Unit Root: How Sure Are We That Economic Time Series Have a Unit Root? Journal of Econometrics, (54), 159-178.

Larrain, M. (1991). Testing Chaos and Nonlinearities in T-bill Rates. Financial Analysts Journal, 47(5), 51-62.

Lee, J. and M. Strazicich (2003). Minimum LM Unit Root Test with Two Structural Breaks. Review of Economics and Statistics, (85), 1082-1089.

Lendasse, A., de Bodt, E., Wertz, V., and Verleysen, M. (2000). Non-Linear Financial Time Series Forecasting Application to the BEL 20 Stock Market Index. European Journal of Economic and Social Systems, 14(1), 81-92.

Lim, K. P., and Brooks, R. (2011). The Evolution of Stock Market Efficiency Over Time: a Survey of the Empirical Literature. Journal of Economic Surveys, 25(1), 69-108.

Lim, K. P., Brooks, R. D., and Hinich, M. J. (2008). Nonlinear serial dependence and the weak-form efficiency of Asian emerging stock markets. Journal of International Financial Markets, Institutions and Money, 18(5), 527-544.

Liow, K.H. and Webb, J.R. (2008).. Nonlinear Return Dependence in Major Real Estate Markets. Journal of Property Research, 25(4), 285-319.

Liljblom, E., and Stenius, M. (1997). Macroeconomic Volatility and Stock Market Volatility: Empirical Evidence on Finnish Data." Applied Financial Economics 7, 419-426.

Lu, C. and So, R.W. (2001). The Relationship between REITs Returns and Inflation: A Vector Error Correction Approach. Review of Quantitative Finance and Accounting, 16(2), 103-115.

Lumsdaine, R. and D. Papell (1997). Multiple Trend Breaks and the Unit Root Hypothesis. Review of Economics and Statistics, (79), 212-218.

McQuenn, G. and Thorley, S. (1991), Are Stock Returns Predictable? A Test Using Markov Chains. Journal of Finance, 46(1), 239-263.

McQueen, G. and Thorley, S. (1994) Bubbles, Stock Returns, and Duration Dependence. Journal of Financial and Quantitative Analysis, 29, 379-401.

Morelli, D. (2002). The Relationship Between Conditional Stock Market Volatility and Conditional Macroeconomic Volatility: Empirical Evidence Based on UK Date. International Review of Financial Analysis, 11, 101-110.

Newell, G. and Matysiak, G (1997). An Empirical Investigation into the Presence of Chaotic Behavior in U.K. Property Market. Research Report. The Royal Institute of Chartered Surveyors, 1997.

Nychka, D., Ellner, S., Gallant, A. R., and McCaffrey, D. (1992). Finding Chaos in Noisy Systems. Journal of the Royal Statistical Society. Series B (Methodological), 399-426.

Odabasi, A., Aksu, C., and Akgiray, V. (2004). The Statistical Evolution of Prices on the Istanbul Stock Exchange. The European Journal of Finance, 10(6), 510-525.

Ozer, G. and Ertokatli, C. (2010). Chaotic Processes of Common Stock Index Returns: An Empirical Examination on Istanbul Stock Exchange (ISE) Market. African Journal of Business Management, 4(6), 1140-1148.

Ozkaya, A. and Ozkaya, R. (2012). Uncovering the chaotic behavior of ISE 100 stock-market index. African Journal of Business Management, 6(7), 2727-2737.

Ozun, A. (1999). Chaos Theory, Non-Linear Behavior in Stock Returns, Thin Trading and Market Efficiency in Emerging Markets: The Case of the Istanbul Stock Exchange. Istanbul Stock Exchange Review, 3(9), 41-74. 
Pesaran, M.H. and Timmermann, A. (1995). Predictability of Stock Returns: Robustness and Economic Significance. Journal of Finance, 50, 1201-1228.

Poshakwale, S. (2002). The Random Walk Hypothesis in the Emerging Indian Stock Market. Journal of Business Finance and Accounting, 2002, 29:9, 1275-99.

Ramsey, J.B. and Rothman, P. (1996). Time Reversibility and Business Cycle Asymmetry. Journal of Money, Credit, and Banking, 28, 1-21.

Schmidt, P. and P. Phillips (1992), LM Test for a Unit Root in the Presence of Deterministic Trends. Oxford Bulletin of Economics and Statistics, 54, 257-287.

Schwert, W.G. (1989). Why Does Stock Market Volatility Change Over Time. Journal of Finance 44, 1368-1388.

Shintani, M., and Linton, O. (2004). Nonparametric Neural Network Estimation of Lyapunov Exponents and a Direct Test for Chaos. Journal of Econometrics, 120(1), 1-33.

Teräsvirta, T. (1994). Specification, Estimation, and Evaluation of Smooth Transition Autoregressive Models. Journal of the American Statistical Association, 89(425), 208-218.

Wolf, A., Swift, J. B., Swinney, H. L., and Vastano, J. A. (1985). Determining Lyapunov Exponents from a Time Series. Physica D: Nonlinear Phenomena, 16(3), 285-317.

Yilanci, V. (2012). Detection of Nonlinear Events in Turkish Stock Market. Journal of Applied Economic Sciences (JAES), 1 (19), 93-96.

Zivot, E. and D. Andrews (1992). Further Evidence on the Great Crash, the Oil Price Shock, and the Unit-Root Hypothesis. Journal of Business and Economic Statistics, 10, 251-70. 
This Page Intentionally Left Blank 